Undergraduate Students, Health and Biomedical Sciences

\title{
Clustering of lifestyle risk factors among Algerian adolescents : Comparison between urban and rural area
}

\author{
Dana Saad ${ }^{1}$, Hissa AlMannai ${ }^{1}$, Fatima Alzahra Yakti ${ }^{1}$
}

Supervised by: Dr. Abdelhamid Kerkadi, Grace Attieh

${ }^{1}$ Human Nutrition Department, College of Health Sciences, Qatar University, Doha, Qatar

\section{Introduction}

- NCDs constitute $20 \%$ in third of the Arab countries and $76 \%$ in Algeria.

- Non communicable diseases (NCDs) such as cardiovascular diseases, diabetes are considered the most leading cause of death globally contributing to $71 \%$ of the total deaths worldwide in 2016

- The prevalence of smoking, sedentary behavior and obesity were high, in both urban and rural areas

- The data of the study was derived from the Global School-based Health Survey (GSHS)

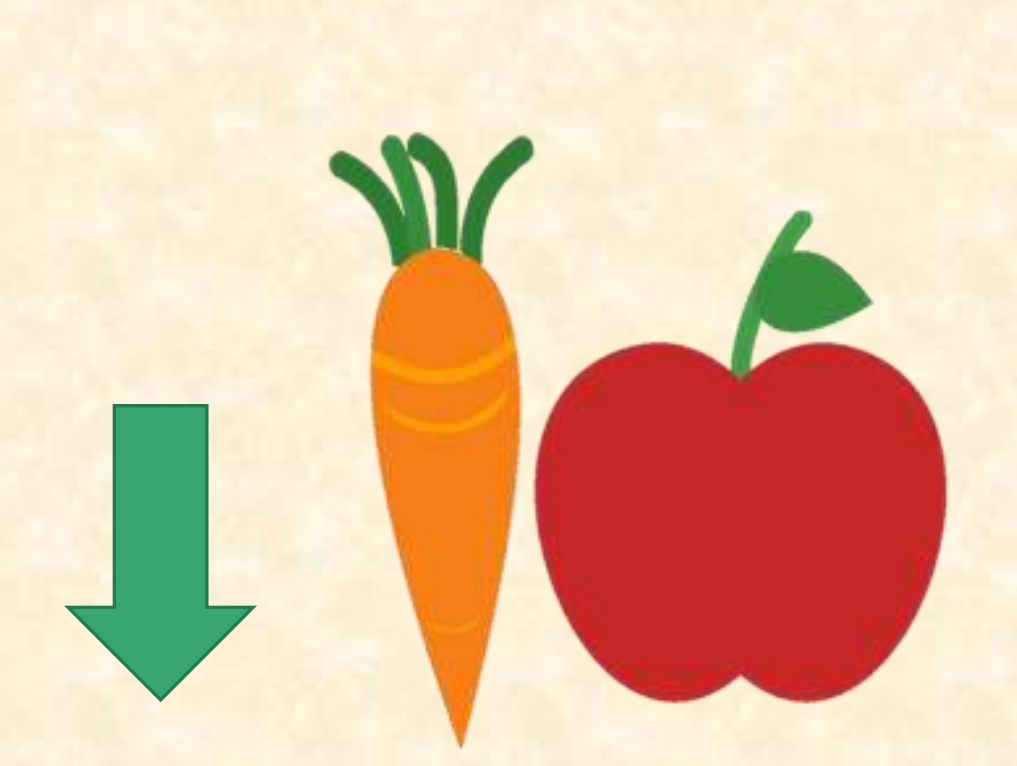

Lifestyle Behaviors risk Factors Studied

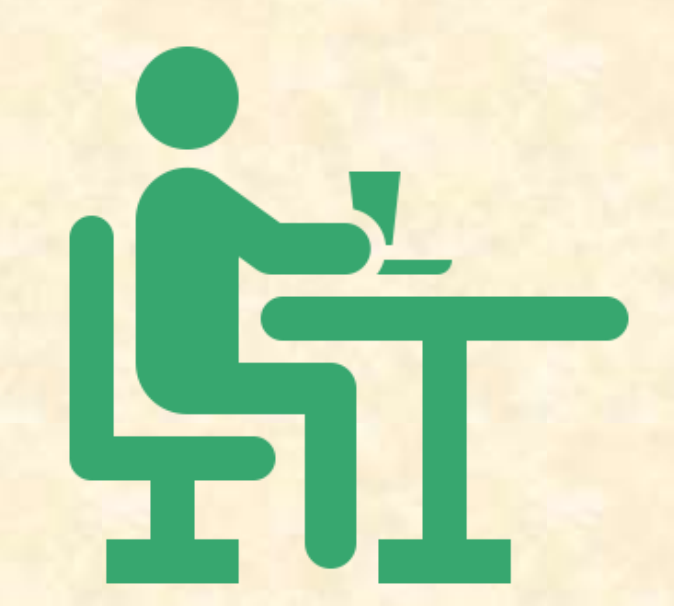

Sedentary behavior

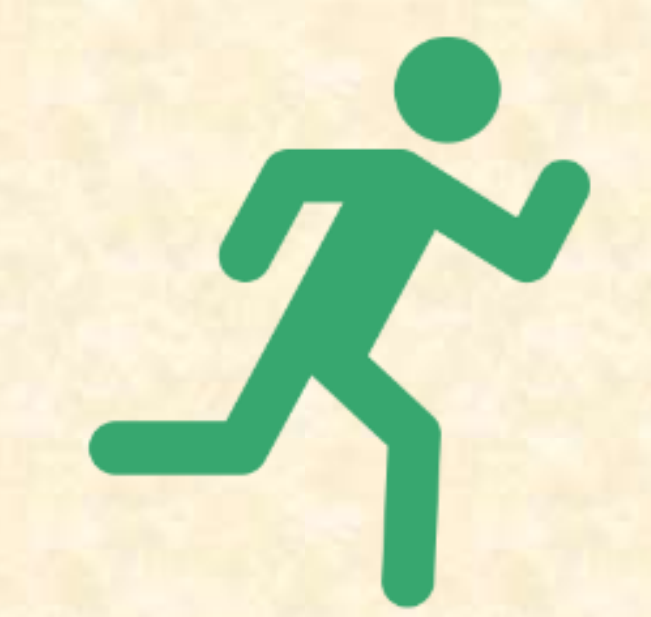

Physical activity Tobacco use

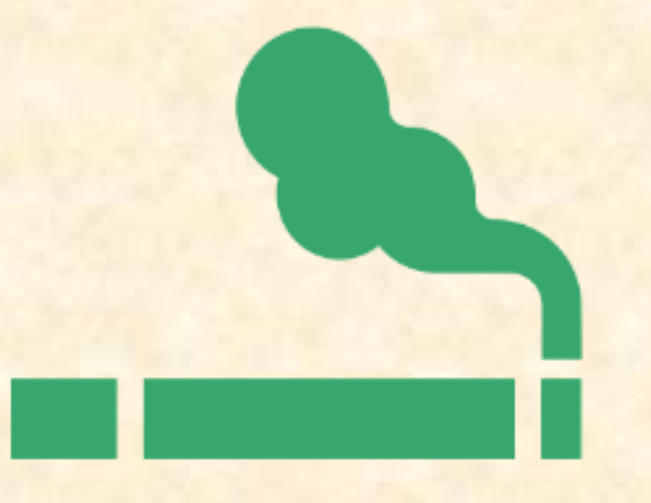

Low Fruits \& Vegetables

Overweight/Obesity

Study Population

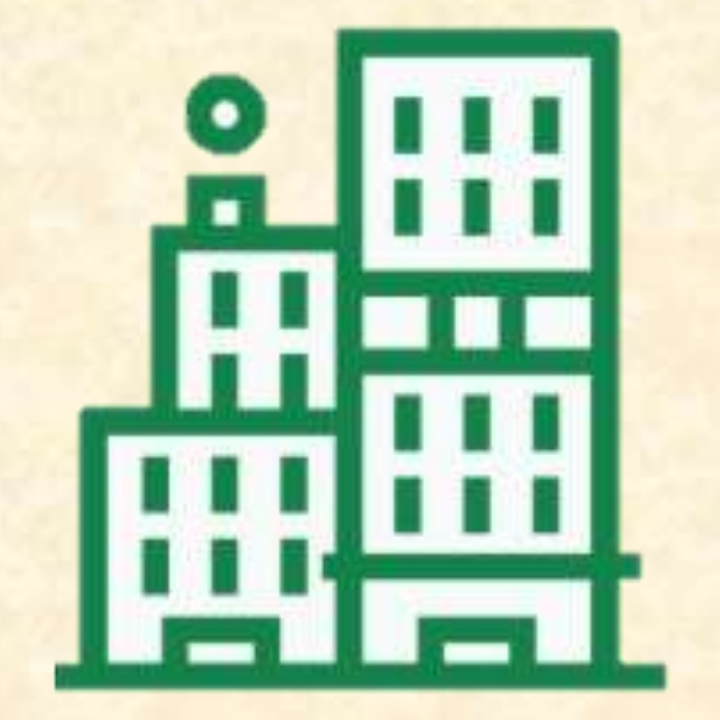

Urban $=2131$

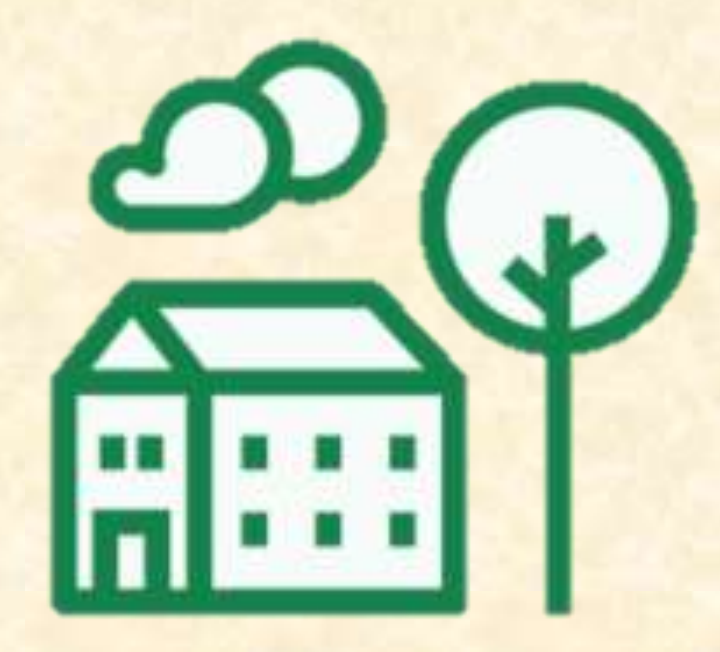

Rural $=2058$

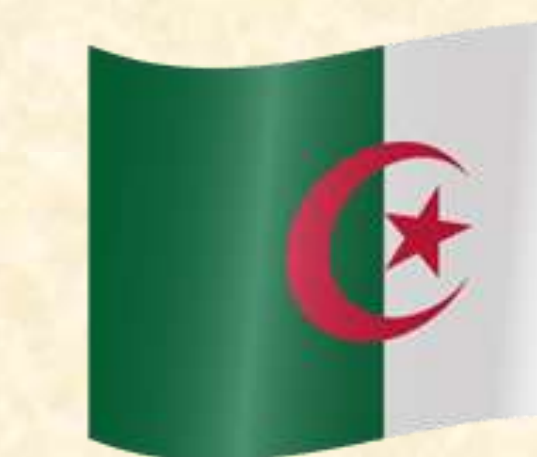

Adolescents aged $11-16$ years old

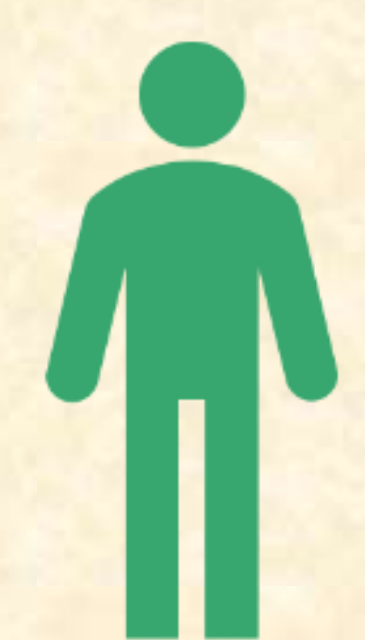

2150

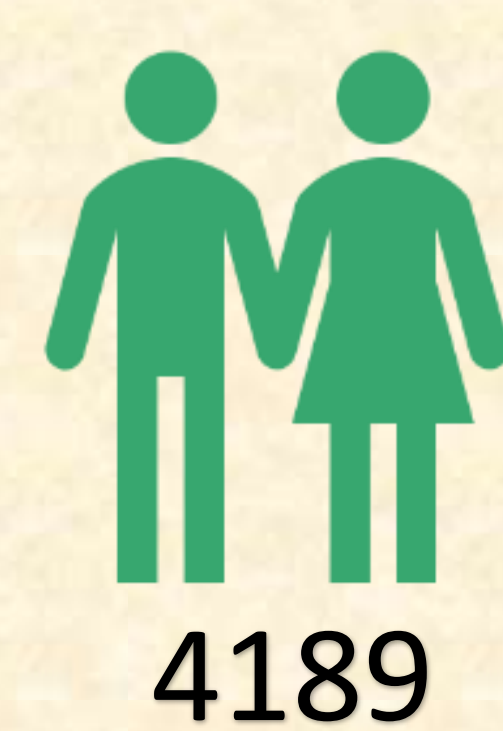

4189

- Clustering of lifestyle factors among adolescents was measured by the ratios of observed (O) and expected (E) prevalence of one or more simultaneously occurring risk behaviours for urban and rural area separately.

- The presence of clustering is identified if the observed-expected ratio $(O / E)$ is higher than one.

- Data were analyzed using SPSS statistical package version 23.

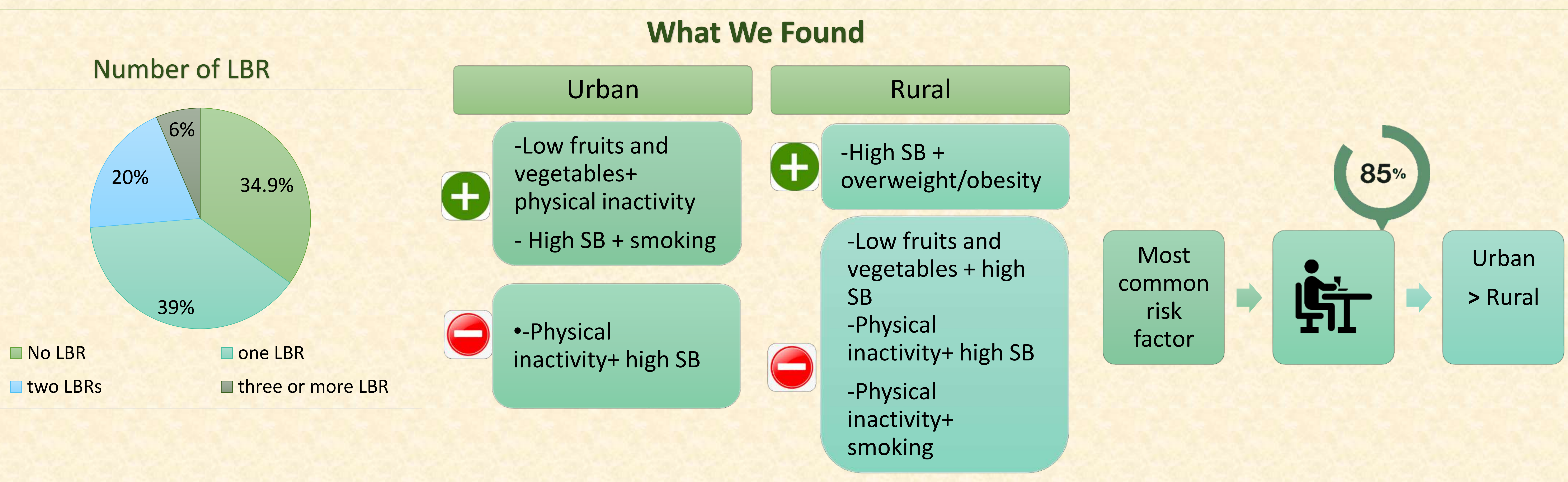

\section{Conclusion}

- Findings from the present study highlighted the high prevalence of multiple lifestyle behavioral risk factors among Algerian adolescents.

- There was a significant difference in the prevalence of LBRs between rural and urban adolescents.

- Several socio-demographic factors (location, age, gender) have been identified to play a role in the LBRs clustering among adolescents in Algeria.

- Further researches including objective measures of LBRs, Socio-economic status indicators, and blood biomarkers are required to understand the importance of LBRs clustering in the development of CVD among adolescents.

- Results of the present study suggest the development of intervention aiming to tackle different LBRs rather than focusing on strategies that address a single LBR.

\section{References}

Algeria - Global School-Based Student Health Survey 2011. Oct 4, 2020. https://extranet.who.int/ncdsmicrodata/index.php/catalog/668

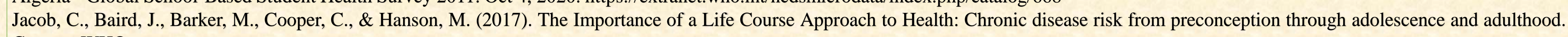
Geneva: WHO.

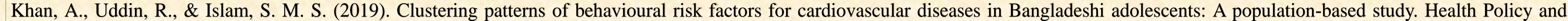
Technology, 8(4), 386-392.

Rocha, F. L., \& Velasquez-Melendez, G. J. E. A. N. (2019). Simultaneity and aggregation of risk factors for noncommunicable diseases among Brazilian adolescents. 23(3). 